\title{
Embedding Theorems for the Dunkl Harmonic Oscillator on the Line
}

\author{
Jesús A. ÁLVAREZ LÓPEZ ${ }^{\dagger}$ and Manuel CALAZA \\ † Departamento de Xeometría e Topoloxía, Facultade de Matemáticas, \\ Universidade de Santiago de Compostela, 15782 Santiago de Compostela, Spain \\ E-mail: jesus.alvarez@usc.es \\ ‡ Laboratorio de Investigación 10, Servicio de Reumatología, Instituto de Investigación \\ Sanitaria, Hospital Clínico Universitario, 15706 Santiago de Compostela, Spain \\ E-mail: manuel.calaza@usc.es
}

Received September 09, 2013, in final form January 06, 2014; Published online January 10, 2014 http://dx.doi.org/10.3842/SIGMA.2014.004

\begin{abstract}
Embedding results of Sobolev type are proved for the Dunkl harmonic oscillator on the line.

Key words: Dunkl harmonic oscillator; Sobolev embedding; generalized Hermite functions; Schwartz space
\end{abstract}

2010 Mathematics Subject Classification: 46E35; 47B25; 33C45

\section{Introduction}

The subindex ev/odd is added to any space of functions on $\mathbb{R}$ to indicate its subspace of even/odd functions; in particular, $C^{\infty}=C_{\mathrm{ev}}^{\infty} \oplus C_{\mathrm{odd}}^{\infty}$ for $C^{\infty}:=C^{\infty}(\mathbb{R})$. The Dunkl operator $T_{\sigma}(\sigma>-1 / 2)$ on $C^{\infty}$ is the perturbation of $\frac{\mathrm{d}}{\mathrm{d} x}$ defined by $T_{\sigma}=\frac{\mathrm{d}}{\mathrm{d} x}$ on $C_{\mathrm{ev}}^{\infty}$ and $T_{\sigma}=\frac{\mathrm{d}}{\mathrm{d} x}+2 \sigma \frac{1}{x}$ on $C_{\mathrm{odd}}^{\infty}$. The corresponding Dunkl harmonic oscillator is the perturbation $L_{\sigma}=-T_{\sigma}^{2}+s^{2} x^{2}$ of the harmonic oscillator $H=-\frac{\mathrm{d}^{2}}{\mathrm{~d} x^{2}}+s^{2} x^{2}(s>0)$. The conjugation $E_{\sigma}=|x|^{\sigma} T_{\sigma}|x|^{-\sigma}$ on $|x|^{\sigma} C^{\infty}$ is equal to $\frac{\mathrm{d}}{\mathrm{d} x}-\sigma x^{-1}$ on $|x|^{\sigma} C_{\mathrm{ev}}^{\infty}$ and $\frac{\mathrm{d}}{\mathrm{d} x}+\sigma x^{-1}$ on $|x|^{\sigma} C_{\text {odd }}^{\infty}$; note that $|x|^{\sigma} C_{\mathrm{ev} / \text { odd }}^{\infty}$ consists of even/odd functions, possibly not smooth or not even defined at 0 . Up to the product by a constant, $E_{\sigma}$ was introduced by Yang [39]. In the form $T_{\sigma}$, this operator was generalized to $\mathbb{R}^{n}$ by Dunkl [12, 13, 14], giving rise to what is now called Dunkl theory (see the survey [31]); in particular, the Dunkl harmonic oscillator on $\mathbb{R}^{n}$ was studied in $[15,25,26,29]$. See [27] for further generalizations on $\mathbb{R}$. Sometimes the terms Yang-Dunkl operator and Yang-Dunkl harmonic oscillator are used in the case of $\mathbb{R}[27]$.

Let $p_{k}$ be the sequence of orthogonal polynomials for the measure $e^{-s x^{2}}|x|^{2 \sigma} \mathrm{d} x$, taken with norm one and positive leading coefficient. Up to normalization, these are the generalized Hermite polynomials [32, p. 380, Problem 25]; see also [9, 10, 11, 16, 29, 30]. The corresponding generalized Hermite functions are $\phi_{k}=p_{k} e^{-s x^{2} / 2}$.

For each $m \in \mathbb{N}$, let $\mathcal{S}^{m}$ be the Banach space of functions $\phi \in C^{m}(\mathbb{R})$ with $\sup _{x}\left|x^{i} \phi^{(j)}(x)\right|<\infty$ for $i+j \leq m$; the corresponding Fréchet space $\mathcal{S}=\bigcap_{m} \mathcal{S}^{m}$ is the Schwartz space on $\mathbb{R}$. With domain $\mathcal{S}, L_{\sigma}$ is essentially self-adjoint in $L^{2}\left(\mathbb{R},|x|^{2 \sigma} \mathrm{d} x\right)$, and the spectrum of its selfadjoint extension, $\mathcal{L}_{\sigma}$, consists of the eigenvalues $(2 k+1+2 \sigma) s(k \in \mathbb{N})$, with corresponding eigenfunctions $\phi_{k}$ [29]. For each real $m \geq 0$, let $W_{\sigma}^{m}$ be the Hilbert space completion of $\mathcal{S}$ with respect to the scalar product $\langle\phi, \psi\rangle_{W_{\sigma}^{m}}:=\left\langle\left(1+\mathcal{L}_{\sigma}\right)^{m} \phi, \psi\right\rangle_{\sigma}$, where $\langle,\rangle_{\sigma}$ denotes the scalar product of $L^{2}\left(\mathbb{R},|x|^{2 \sigma} \mathrm{d} x\right)$, obtaining a Fréchet space $W_{\sigma}^{\infty}=\bigcap_{m} W_{\sigma}^{m}$. We show the following embedding theorems; the second one is of Sobolev type. 
Theorem 1.1. For each $m \in \mathbb{N}, \mathcal{S}_{\text {ev/odd }}^{M_{m, \text { ev dd }}} \subset W_{\sigma, \text { ev /odd }}^{m}$ continuously, where

$$
\begin{aligned}
& M_{m, \mathrm{ev} / \mathrm{odd}}= \begin{cases}\frac{3 m+3}{2}+\frac{m+1}{4}\lceil\sigma\rceil(\lceil\sigma\rceil+3)+\lceil\sigma\rceil & \text { if } \sigma \geq 0 \text { and } m \text { is odd, } \\
2 m+3 & \text { if } \sigma<0 \text { and } m \text { is odd, }\end{cases} \\
& M_{m, \mathrm{ev}}= \begin{cases}\frac{3 m+2}{2}+\frac{m}{4}\lceil\sigma\rceil(\lceil\sigma\rceil+3)+\lceil\sigma\rceil & \text { if } \sigma \geq 0 \text { and } m \text { is even, } \\
2 m+2 & \text { if } \sigma<0 \text { and } m \text { is even, }\end{cases} \\
& M_{m, \text { odd }}= \begin{cases}\frac{3 m+4}{2}+\frac{m+2}{4}\lceil\sigma\rceil(\lceil\sigma\rceil+3)+\lceil\sigma\rceil & \text { if } \sigma \geq 0 \text { and } m \text { is even, } \\
2 m+4 & \text { if } \sigma<0 \text { and } m \text { is even. }\end{cases}
\end{aligned}
$$

Theorem 1.2. For all $m \in \mathbb{N}$ and $m_{\sigma}=m+1+\frac{1}{2}\lceil\sigma\rceil(\lceil\sigma\rceil+1), W_{\sigma \text {,ev/odd }}^{m^{\prime}} \subset \mathcal{S}_{\text {ev/odd }}^{m}$ continuously if $m^{\prime}>N_{m, \mathrm{ev} / \mathrm{odd}}$, where $N_{m, \mathrm{ev}}=2$ and $N_{m, \mathrm{odd}}=5$ if $m_{\sigma}=1, N_{m, \mathrm{ev}}=6$ and $N_{m, \mathrm{odd}}=5$ if $m_{\sigma}=2, N_{m, \mathrm{ev}}=6$ and $N_{m, \mathrm{odd}}=7$ if $m_{\sigma}=3$, and $N_{m, \mathrm{ev} / \mathrm{odd}}=m_{\sigma}+3$ for $m_{\sigma} \geq 4$.

Corollary 1.3. $\mathcal{S}=W_{\sigma}^{\infty}$ as Fréchet spaces.

In other words, Corollary 1.3 states that an element $\phi \in L^{2}\left(\mathbb{R},|x|^{2 \sigma} \mathrm{d} x\right)$ is in $\mathcal{S}$ if and only if the "Fourier coefficients" $\left\langle\phi, \phi_{k}\right\rangle_{\sigma}$ are rapidly decreasing on $k$. This also means that $\mathcal{S}=\bigcap_{m} \mathcal{D}\left(\mathcal{L}_{\sigma}^{m}\right)$ ( $\mathcal{S}$ is the smooth core of $\mathcal{L}_{\sigma}$ with the terminology of [6]) because the sequence of eigenvalues of $\mathcal{L}_{\sigma}$ is in $O(k)$ as $k \rightarrow \infty$.

We introduce a version $\mathcal{S}_{\sigma}^{m}$ of every $\mathcal{S}^{m}$, whose definition involves $T_{\sigma}$ instead of $\frac{\mathrm{d}}{\mathrm{d} x}$. They satisfy much simpler embeddings: $S_{\sigma}^{\lceil m\rceil+1} \subset W_{\sigma}^{m}$, and $W_{\sigma}^{m^{\prime}} \subset \mathcal{S}_{\sigma}^{m}$ if $m^{\prime}-m>1$. Even though $\mathcal{S}=\bigcap_{m} \mathcal{S}_{\sigma}^{m}$, the inclusion relations between the spaces $\mathcal{S}_{\sigma}^{m}$ and $\mathcal{S}^{m^{\prime}}$ are complicated, giving rise to the complexity of Theorems 1.1 and 1.2.

Other Sobolev type embedding theorems, for different operators and with different techniques, were recently proved in $[35,36,37]$.

Next, we consider other perturbations of $H$ on $\mathbb{R}_{+}$. Let $\mathcal{S}_{\text {ev }, U}$ denote the space of restrictions of even Schwartz functions to some open $U \subset \mathbb{R}_{+}$, and set $\phi_{k, U}=\left.\phi_{k}\right|_{U}$.

Theorem 1.4. Let $P=H-2 f_{1} \frac{\mathrm{d}}{\mathrm{d} x}+f_{2}$, where $f_{1} \in C^{1}(U)$ and $f_{2} \in C(U)$ for some open $U \subset \mathbb{R}_{+}$of full Lebesgue measure. Assume that $f_{2}=\sigma(\sigma-1) x^{-2}-f_{1}^{2}-f_{1}^{\prime}$ for some $\sigma>-1 / 2$. Let $h=x^{\sigma} e^{-F_{1}}$, where $F_{1} \in C^{2}(U)$ is a primitive of $f_{1}$. Then the following properties hold:

(i) $P$, with domain $h \mathcal{S}_{\mathrm{ev}, U}$, is essentially self-adjoint in $L^{2}\left(U, e^{2 F_{1}} \mathrm{~d} x\right)$;

(ii) the spectrum of its self-adjoint extension, $\mathcal{P}$, consists of the eigenvalues $(4 k+1+2 \sigma) s$ $(k \in \mathbb{N})$ with multiplicity one and normalized eigenfunctions $\sqrt{2} h \phi_{2 k, U}$; and

(iii) the smooth core of $\mathcal{P}$ is $h \mathcal{S}_{\mathrm{ev}, U}$.

This theorem follows by showing that the stated condition on $f_{1}$ and $f_{2}$ characterizes the cases where $P$ can be obtained by the following process: first, restricting $L_{\sigma}$ to even functions, then restricting to $U$, and finally conjugating by $h$. The term of $P$ with $\frac{\mathrm{d}}{\mathrm{d} x}$ can be removed by conjugation with the product of a positive function, obtaining the operator $H+\sigma(\sigma-1) x^{-2}$; in this way, we get all operators of the form $H+c x^{-2}$ with $c>-1 / 4$.

The conditions of Theorem 1.4 are satisfied by $P=H-2 c_{1} x^{-1} \frac{\mathrm{d}}{\mathrm{d} x}+c_{2} x^{-2}\left(c_{1}, c_{2} \in \mathbb{R}\right)$ on $\mathbb{R}_{+}$ if and only if there is some $a \in \mathbb{R}$ such that $a^{2}+\left(2 c_{1}-1\right) a-c_{2}=0$ and $a+c_{1}>-1 / 2$; in this case, $h=x^{a}$ and $e^{2 F_{1}}=x^{2 c_{1}}$. For some $c_{1}, c_{2} \in \mathbb{R}$, there are two values of $a$ satisfying these conditions, obtaining two different self-adjoint operators defined by $P$ in different Hilbert spaces. For instance, $L_{\sigma}$ may define a self-adjoint operator when $\sigma \leq-1 / 2$. 
This example is applied in [2] to prove a new type of Morse inequalities on strata of compact stratifications [20, 33, 34] with adapted metrics [5, 23, 24], where the Witten's perturbation [38] is used for the minimal/maximal ideal boundary conditions of de Rham complex $[6,7,8]$. The version of Morse functions used in [2] is different from the version of Goresky-MacPherson [18]. More precisely, in the local conic model of a stratification around each critical point, by induction on the depth of the stratification, it is assumed that the Laplacian of the minimal/maximal boundary condition of the de Rham complex of the link (section of the cone) has a nice spectral decomposition. Using this, the Witten's perturbation of the de Rham complex of the cone splits into an infinite direct sum of elliptic complexes of two simple types, with length one and two, which represent the radial direction of Witten's perturbed complex. It turns out that the Witten's perturbed Laplacian of these simple complexes is described by the above operator $P$, and the two possible choices of the constant $a$ give rise to the minimal/maximal ideal boundary conditions. In this way, Theorem 1.4 becomes a key ingredient of [2].

\section{Preliminaries}

\subsection{Dunkl operator on the line}

For any $\phi \in C^{\infty}:=C^{\infty}(\mathbb{R})$, there exists some $\psi \in C^{\infty}$ so that $\phi(x)-\phi(0)=x \psi(x)$; moreover

$$
\psi^{(m)}(x)=\int_{0}^{1} t^{m} \phi^{(m+1)}(t x) \mathrm{d} t
$$

for all $^{1} m \in \mathbb{N}$ (see e.g. [19, Theorem 1.1.9]). Let us use the notation $\psi=x^{-1} \phi$. The Dunkl operator on $T_{\sigma}(\sigma \in \mathbb{R})$ on $C^{\infty}$ is the perturbation of $\frac{\mathrm{d}}{\mathrm{d} x}$ defined by

$$
\left(T_{\sigma} \phi\right)(x)=\phi^{\prime}(x)+2 \sigma \frac{\phi(x)-\phi(-x)}{x} .
$$

Consider matrix expressions of operators on $C^{\infty}$ with respect to the decomposition $C^{\infty}=$ $C_{\mathrm{ev}}^{\infty} \oplus C_{\mathrm{odd}}^{\infty}$, as direct sum of subspaces of even and odd functions. For each function $h$, the notation $h$ is also used for the operator of multiplication by $h$. Then

$$
\begin{aligned}
\frac{\mathrm{d}}{\mathrm{d} x} & =\left(\begin{array}{cc}
0 & \frac{\mathrm{d}}{\mathrm{d} x} \\
\frac{\mathrm{d}}{\mathrm{d} x} & 0
\end{array}\right), \quad x=\left(\begin{array}{cc}
0 & x \\
x & 0
\end{array}\right), \\
T_{\sigma} & =\left(\begin{array}{cc}
0 & \frac{\mathrm{d}}{\mathrm{d} x}+2 \sigma x^{-1} \\
\frac{\mathrm{d}}{\mathrm{d} x} & 0
\end{array}\right)=\frac{\mathrm{d}}{\mathrm{d} x}+2 \sigma\left(\begin{array}{cc}
0 & x^{-1} \\
0 & 0
\end{array}\right)
\end{aligned}
$$

on $C^{\infty}$. With $\Sigma=\left(\begin{array}{cc}\sigma & 0 \\ 0 & -\sigma\end{array}\right)$, we get

$$
\left[T_{\sigma}, x\right]=1+2 \Sigma, \quad T_{\sigma} \Sigma+\Sigma T_{\sigma}=x \Sigma+\Sigma x=0 .
$$

\subsection{Dunkl harmonic oscillator on the line}

On $C^{\infty}$, the harmonic oscillator, and the annihilation and creation operators are $H=-\frac{\mathrm{d}^{2}}{\mathrm{~d} x^{2}}+$ $s^{2} x^{2}, A=s x+\frac{\mathrm{d}}{\mathrm{d} x}$ and $A^{\prime}=s x-\frac{\mathrm{d}}{\mathrm{d} x}(s>0)$. Their perturbations $L=-T_{\sigma}^{2}+s^{2} x^{2}, B=s x+T_{\sigma}$

\footnotetext{
${ }^{1}$ We adopt the convention $0 \in \mathbb{N}$.
} 
and $B^{\prime}=s x-T_{\sigma}$ are called Dunkl harmonic oscillator, and Dunkl annihilation and creation operators. By (2),

$$
\begin{aligned}
& L=B B^{\prime}-(1+2 \Sigma) s=B^{\prime} B+(1+2 \Sigma) s=\frac{1}{2}\left(B B^{\prime}+B^{\prime} B\right), \\
& {[L, B]=-2 s B, \quad\left[L, B^{\prime}\right]=2 s B^{\prime}} \\
& {\left[B, B^{\prime}\right]=2 s(1+2 \Sigma),} \\
& {[L, \Sigma]=B \Sigma+\Sigma B=B^{\prime} \Sigma+\Sigma B^{\prime}=0 .}
\end{aligned}
$$

For each $m \in \mathbb{N}$, let $\mathcal{S}^{m}$ be the space of functions $\phi \in C^{\infty}$ such that

$$
\|\phi\|_{\mathcal{S}^{m}}=\sum_{i+j \leq m} \sup _{x}\left|x^{i} \phi^{(j)}(x)\right|<\infty
$$

This expression defines a norm \|\|$_{\mathcal{S}^{m}}$ on $\mathcal{S}^{m}$, which becomes a Banach space. We have $\mathcal{S}^{m+1} \subset \mathcal{S}^{m}$ continuously ${ }^{2}$, and $\mathcal{S}=\bigcap_{m} \mathcal{S}^{m}$, with the induced Fréchet topology, is the Schwartz space on $\mathbb{R}$. Note that $\left\|\phi^{\prime}\right\|_{\mathcal{S}^{m}} \leq\|\phi\|_{\mathcal{S}^{m+1}}$ for all $m$.

We can restrict the above decomposition of $C^{\infty}$ to every $\mathcal{S}^{m}$ and $\mathcal{S}$, obtaining $\mathcal{S}^{m}=\mathcal{S}_{\text {ev }}^{m} \oplus \mathcal{S}_{\text {odd }}^{m}$ and $\mathcal{S}=\mathcal{S}_{\text {ev }} \oplus \mathcal{S}_{\text {odd }}$. The matrix expressions of operators on $\mathcal{S}$ are taken with respect to this decomposition. For $\phi \in C_{\mathrm{ev}}^{\infty}, \psi=x^{-1} \phi$ and $i, j \in \mathbb{N}$, we get from (1) that

$$
\left|x^{i} \psi^{(j)}(x)\right| \leq \int_{0}^{1} t^{j-i}\left|(t x)^{i} \phi^{(j+1)}(t x)\right| \mathrm{d} t \leq \sup _{y \in \mathbb{R}}\left|y^{i} \phi^{(j+1)}(y)\right|
$$

for all $x \in \mathbb{R}$. So $\|\psi\|_{\mathcal{S}^{m}} \leq\|\phi\|_{\mathcal{S}^{m+1}}$ for all $m \in \mathbb{N}$, obtaining that $\mathcal{S}_{\text {odd }}=x \mathcal{S}_{\text {ev }}$ and $x^{-1}$ : $C_{\text {odd }}^{\infty} \rightarrow C_{\mathrm{ev}}^{\infty}$ restricts to a continuous operator $x^{-1}: \mathcal{S}_{\text {odd }} \rightarrow \mathcal{S}_{\text {ev }}$. Hence $x: \mathcal{S}_{\text {ev }} \rightarrow \mathcal{S}_{\text {odd }}$ is an isomorphism of Fréchet spaces, and $T_{\sigma}, B, B^{\prime}$ and $L$ define continuous operators on $\mathcal{S}$.

Let $\langle,\rangle_{\sigma}$ and \|\|$_{\sigma}$ be the scalar product and norm of $L^{2}\left(\mathbb{R},|x|^{2 \sigma} \mathrm{d} x\right)$. Suppose from now on that $\sigma>-1 / 2$, obtaining that $\mathcal{S}$ is dense in $L^{2}\left(\mathbb{R},|x|^{2 \sigma} \mathrm{d} x\right)$. The following properties hold considering these operators in $L^{2}\left(\mathbb{R},|x|^{2 \sigma} \mathrm{d} x\right)$ with domain $\mathcal{S}:-T_{\sigma}$ is adjoint of $T_{\sigma}, B^{\prime}$ is adjoint of $B$, and $L$ is essentially self-adjoint. Let $\mathcal{L}$, or $\mathcal{L}_{\sigma}$, denote the self-adjoint extension of $L$ (with domain $\mathcal{S})$. Its spectrum consists of the eigenvalues $(2 k+1+2 \sigma) s(k \in \mathbb{N})$. The corresponding normalized eigenfunctions $\phi_{k}$ are inductively defined by

$$
\begin{aligned}
\phi_{0} & =s^{(2 \sigma+1) / 4} \Gamma(\sigma+1 / 2)^{-1 / 2} e^{-s x^{2} / 2}, \\
\phi_{k} & =\left\{\begin{array}{ll}
(2 k s)^{-1 / 2} B^{\prime} \phi_{k-1} & \text { if } k \text { is even, } \\
(2(k+2 \sigma) s)^{-1 / 2} B^{\prime} \phi_{k-1} & \text { if } k \text { is odd, }
\end{array} \quad k \geq 1 .\right.
\end{aligned}
$$

Furthermore

$$
\begin{aligned}
& B \phi_{0}=0 \\
& B \phi_{k}=\left\{\begin{array}{ll}
(2 k s)^{1 / 2} \phi_{k-1} & \text { if } k \text { is even, } \\
(2(k+2 \sigma) s)^{1 / 2} \phi_{k-1} & \text { if } k \text { is odd, }
\end{array} \quad k \geq 1 .\right.
\end{aligned}
$$

These properties follow from (3)-(6), like in the case of $H$.

\footnotetext{
${ }^{2}$ For topological vector spaces $X$ and $Y$, it is said that $X \subset Y$ continuously if $X$ is a linear subspace of $Y$ and the inclusion map $X \hookrightarrow Y$ is continuous.
} 


\subsection{Generalized Hermite polynomials}

By (7), (8) and the definition of $B^{\prime}$, we get $\phi_{k}=p_{k} e^{-s x^{2} / 2}$, where $p_{k}$ is the sequence of polynomials inductively given by $p_{0}=s^{(2 \sigma+1) / 4} \Gamma(\sigma+1 / 2)^{-1 / 2}$ and

$$
p_{k}=\left\{\begin{array}{ll}
(2 k s)^{-1 / 2}\left(2 \operatorname{sxp}_{k-1}-T_{\sigma} p_{k-1}\right) & \text { if } k \text { is even, } \\
(2(k+2 \sigma) s)^{-1 / 2}\left(2 s x p_{k-1}-T_{\sigma} p_{k-1}\right) & \text { if } k \text { is odd, }
\end{array} \quad k \geq 1 .\right.
$$

Up to normalization, $p_{k}$ and $\phi_{k}$ are the generalized Hermite polynomials and functions [32, p. 380, Problem 25]. Each $p_{k}$ is of degree $k$, even/odd if $k$ is even/odd, and with positive leading coefficient. Moreover $T_{\sigma} p_{0}=0$ and

$$
T_{\sigma} p_{k}=\left\{\begin{array}{ll}
(2 k s)^{1 / 2} p_{k-1} & \text { if } k \text { is even, } \\
(2(k+2 \sigma) s)^{1 / 2} p_{k-1} & \text { if } k \text { is odd, }
\end{array} \quad k \geq 1 .\right.
$$

From (11) and (12), we obtain the recursion formula

$$
p_{k}= \begin{cases}k^{-1 / 2}\left((2 s)^{1 / 2} x p_{k-1}-(k-1+2 \sigma)^{1 / 2} p_{k-2}\right) & \text { if } k \text { is even, } \\ (k+2 \sigma)^{-1 / 2}\left((2 s)^{1 / 2} x p_{k-1}-(k-1)^{1 / 2} p_{k-2}\right) & \text { if } k \text { is odd. }\end{cases}
$$

By (13) and induction on $k$, we easily get the following when $k$ is $\operatorname{odd}^{3}$ :

$$
x^{-1} p_{k}=\sum_{\ell \in\{0,2, \ldots, k-1\}}(-1)^{\frac{k-\ell-1}{2}} \sqrt{\frac{(k-1)(k-3) \cdots(\ell+2) 2 s}{(k+2 \sigma)(k-2+2 \sigma) \cdots(\ell+1+2 \sigma)}} p_{\ell} .
$$

The following theorem contains a simplified version of the asymptotic estimates satisfied by $\phi_{k}$ and $\xi_{k}=|x|^{\sigma} \phi_{k}$. They can be obtained by expressing $p_{n}$ in terms of the Laguerre polynomials [30, 31], whose asymptotic estimates are studied in [3, 17, 21, 22]. The method of Bonan-Clark [4] can be also applied [1].

Theorem 2.1. There exist $C, C^{\prime}, C^{\prime \prime}>0$, depending on $\sigma$ and $s$, such that:

(i) if $k$ is odd or $\sigma \geq 0$, then $\xi_{k}^{2}(x) \leq C^{\prime} k^{-1 / 6}$ for all $x \in \mathbb{R}$;

(ii) if $k$ is even and positive, and $\sigma<0$, then $\xi_{k}^{2}(x) \leq C^{\prime \prime} k^{-1 / 6}$ for $|x| \geq 1$; and

(iii) if $\sigma<0$, then $\phi_{k}^{2}(x) \leq C^{\prime \prime}$ for all $k$ and $|x| \leq 1$.

\section{$3 \quad$ Perturbed Schwartz space}

We introduce a perturbed version $\mathcal{S}_{\sigma}^{m}$ of each $\mathcal{S}^{m}$ that will be appropriate to show our embedding results. Since $\mathcal{S}_{\sigma}^{m}$ must contain the functions $\phi_{k}$, Theorem 2.1 indicates that different definitions must be given for $\sigma \geq 0$ and $\sigma<0$.

When $\sigma \geq 0$, for any $\phi \in C^{\infty}$ and $m \in \mathbb{N}$, let

$$
\|\phi\|_{\mathcal{S}_{\sigma}^{m}}=\sum_{i+j \leq m} \sup _{x}|x|^{\sigma}\left|x^{i} T_{\sigma}^{j} \phi(x)\right| .
$$

This defines a norm \|\|$_{\mathcal{S}_{\sigma}^{m}}$ on the linear space of functions $\phi \in C^{\infty}$ with $\|\phi\|_{\mathcal{S}_{\sigma}^{m}}<\infty$, and let $\mathcal{S}_{\sigma}^{m}$ denote the corresponding Banach space completion. There are direct sum decompositions into subspaces of even and odd functions, $\mathcal{S}_{\sigma}^{m}=\mathcal{S}_{\sigma, \text { ev }}^{m} \oplus \mathcal{S}_{\sigma, \text { odd }}^{m}$.

\footnotetext{
${ }^{3}$ As a convention, the product of an empty set of factors is 1 . Thus $(k-1)(k-3) \cdots(\ell+2)=1$ for $\ell=k-1$ in (14).
} 
When $\sigma<0$, the even and odd functions are considered separately: let

$$
\begin{aligned}
\|\phi\|_{\mathcal{S}_{\sigma}^{m}}= & \sum_{i+j \leq m, i+j \text { even }}\left(\sup _{|x| \leq 1}\left|x^{i}\left(T_{\sigma}^{j} \phi\right)(x)\right|+\sup _{|x| \geq 1}|x|^{\sigma}\left|x^{i}\left(T_{\sigma}^{j} \phi\right)(x)\right|\right) \\
& +\sum_{i+j \leq m, i+j \text { odd }} \sup _{x \neq 0}|x|^{\sigma}\left|x^{i}\left(T_{\sigma}^{j} \phi\right)(x)\right|
\end{aligned}
$$

for $\phi \in C_{\mathrm{ev}}^{\infty}$, and

$$
\begin{aligned}
\|\phi\|_{\mathcal{S}_{\sigma}^{m}}= & \sum_{i+j \leq m, i+j \text { even }} \sup _{x \neq 0}|x|^{\sigma}\left|x^{i}\left(T_{\sigma}^{j} \phi\right)(x)\right| \\
& +\sum_{i+j \leq m, i+j \text { odd }}\left(\sup _{|x| \leq 1}\left|x^{i}\left(T_{\sigma}^{j} \phi\right)(x)\right|+\sup _{|x| \geq 1}|x|^{\sigma}\left|x^{i}\left(T_{\sigma}^{j} \phi\right)(x)\right|\right)
\end{aligned}
$$

for $\phi \in C_{\mathrm{odd}}^{\infty}$. These expressions define a norm \|\|$_{\mathcal{S}_{\sigma}^{m}}$ on the linear spaces of functions $\phi \in C_{\mathrm{ev} / \mathrm{odd}}^{\infty}$ with $\|\phi\|_{\mathcal{S}_{\sigma}^{m}}<\infty$. The corresponding Banach space completions will be denoted by $\mathcal{S}_{\sigma, \mathrm{ev} / \text { odd }}^{m}$, and let $\mathcal{S}_{\sigma}^{m}=\mathcal{S}_{\sigma, \text { ev }}^{m} \oplus \mathcal{S}_{\sigma, \text { odd }}^{m}$.

In any case, there are continuous inclusions $\mathcal{S}_{\sigma}^{m+1} \subset \mathcal{S}_{\sigma}^{m}$, and a perturbed Schwartz space is defined as $\mathcal{S}_{\sigma}=\bigcap_{m} \mathcal{S}_{\sigma}^{m}$, with the corresponding Fréchet topology, which decomposes as direct sum of the subspaces of even and odd functions, $\mathcal{S}_{\sigma}=\mathcal{S}_{\sigma, \text { ev }} \oplus \mathcal{S}_{\sigma \text {,odd }}$; in particular, $\mathcal{S}_{0}=\mathcal{S}$. It easily follows that $\mathcal{S}_{\sigma}$ consists of functions that are $C^{\infty}$ on $\mathbb{R} \backslash\{0\}$ but a priori possibly not even defined at zero, and $\mathcal{S}_{\sigma}^{m} \cap C^{\infty}$ is dense in $\mathcal{S}_{\sigma}^{m}$ for all $m$; thus $\mathcal{S}_{\sigma} \cap C^{\infty}$ is dense in $\mathcal{S}_{\sigma}$.

Obviously, $\Sigma$ defines a bounded operator on each $\mathcal{S}_{\sigma}^{m}$. It is also easy to see that $T_{\sigma}$ defines a bounded operator $\mathcal{S}_{\sigma}^{m+1} \rightarrow \mathcal{S}_{\sigma}^{m}$ for any $m$; notice that, when $\sigma<0$, the role played by the parity of $i+j$ fits well to prove this property. Similarly, $x$ defines a bounded operator $\mathcal{S}_{\sigma}^{m+1} \rightarrow \mathcal{S}_{\sigma}^{m}$ for any $m$ because, by $(2)$,

$$
\left[T_{\sigma}^{j}, x\right]= \begin{cases}j T_{\sigma}^{j-1} & \text { if } j \text { is even } \\ (j+2 \Sigma) T_{\sigma}^{j-1} & \text { if } j \text { is odd. }\end{cases}
$$

So $B$ and $B^{\prime}$ define bounded operators $\mathcal{S}_{\sigma}^{m+1} \rightarrow \mathcal{S}_{\sigma}^{m}$, and $L$ a bounded operator $\mathcal{S}_{\sigma}^{m+2} \rightarrow \mathcal{S}_{\sigma}^{m}$. Thus $T_{\sigma}, x, \Sigma, B, B^{\prime}$ and $L$ define continuous operators on $\mathcal{S}_{\sigma}$.

In order to prove Theorems 1.1 and 1.2, we introduce an intermediate weakly perturbed Schwartz space $\mathcal{S}_{w, \sigma}$. Like $\mathcal{S}_{\sigma}$, it is a Fréchet space of the form $\mathcal{S}_{w, \sigma}=\bigcap_{m} \mathcal{S}_{w, \sigma}^{m}$, where each $\mathcal{S}_{w, \sigma}^{m}$ is the Banach space defined like $\mathcal{S}_{\sigma}^{m}$ by using $\frac{\mathrm{d}}{\mathrm{d} x}$ instead of $T_{\sigma}$ in the right hand sides of (15)-(17); in particular, $\mathcal{S}_{w, \sigma}^{0}=\mathcal{S}_{\sigma}^{0}$ as Banach spaces. Let \|\|$_{\mathcal{S}_{w, \sigma}^{m}}$ denote the norm of $\mathcal{S}_{w, \sigma}^{m}$. As before, $\mathcal{S}_{w, \sigma}$ consists of functions which are $C^{\infty}$ on $\mathbb{R} \backslash\{0\}$ but a priori possibly not even defined at zero, $\mathcal{S}_{w, \sigma} \cap C^{\infty}$ is dense in $\mathcal{S}_{w, \sigma}$, there is a canonical decomposition $\mathcal{S}_{w, \sigma}=\mathcal{S}_{w, \sigma, \mathrm{ev}} \oplus \mathcal{S}_{w, \sigma, \mathrm{odd}}$, and $\frac{\mathrm{d}}{\mathrm{d} x}$ and $x$ define bounded operators on $\mathcal{S}_{w, \sigma}^{m+1} \rightarrow \mathcal{S}_{w, \sigma}^{m}$. Thus $\frac{\mathrm{d}}{\mathrm{d} x}$ and $x$ define continuous operators on $\mathcal{S}_{w, \sigma}$.

Lemma 3.1. If $\sigma \geq 0$, then $\mathcal{S}^{m+\lceil\sigma\rceil} \subset \mathcal{S}_{w, \sigma}^{m}$ continuously.

Proof. Let $\phi \in \mathcal{S}$. For all $i$ and $j$, we have $|x|^{\sigma}\left|x^{i} \phi^{(j)}(x)\right| \leq\left|x^{i+\lceil\sigma\rceil} \phi^{(j)}(x)\right|$ for $|x| \geq 1$, and $|x|^{\sigma}\left|x^{i} \phi^{(j)}(x)\right| \leq\left|x^{i} \phi^{(j)}(x)\right|$ for $|x| \leq 1$. So $\|\phi\|_{\mathcal{S}_{w, \sigma}^{m}} \leq\|\phi\|_{\mathcal{S}^{m+\lceil\sigma\rceil}}$.

Lemma 3.2. If $\sigma \geq 0, \mathcal{S}_{w, \sigma}^{m_{\sigma}} \subset \mathcal{S}^{m}$ continuously, where $m_{\sigma}=m+1+\frac{1}{2}\lceil\sigma\rceil(\lceil\sigma\rceil+1)$.

Proof. Let $\phi \in \mathcal{S}_{w, \sigma}$. For all $i$ and $j$,

$$
\left|x^{i} \phi^{(j)}(x)\right| \leq|x|^{\sigma}\left|x^{i} \phi^{(j)}(x)\right|
$$

for $|x| \geq 1$. It remains to prove an inequality of this type for $|x| \leq 1$, which will be a consequence of the following assertion. 
Claim 1. For each $n \in \mathbb{N}$, there are finite families of real numbers, $c_{a, b}^{n}, d_{k, \ell}^{n}$ and $e_{u, v}^{n}$, where the indices $a, b, k, \ell, u$ and $v$ run in finite subsets of $\mathbb{N}$ with $b, \ell, v \leq M_{n}=1+\frac{n(n+1)}{2}$ and $k \geq n$, such that, for all $\phi \in C^{\infty}$,

$$
\phi(x)=\sum_{a, b} c_{a, b}^{n} x^{a} \phi^{(b)}(1)+\sum_{k, \ell} d_{k, \ell}^{n} x^{k} \phi^{(\ell)}(x)+\sum_{u, v} e_{u, v}^{n} x^{u} \int_{x}^{1} t^{n} \phi^{(v)}(t) \mathrm{d} t .
$$

Assuming that Claim 1 is true, the proof can be completed as follows. Let $\phi \in \mathcal{S}_{w, \sigma}$ and set $n=\lceil\sigma\rceil$. For $|x| \leq 1$, according to Claim $1,|\phi(x)|$ is bounded by

$$
\begin{aligned}
& \sum_{a, b}\left|c_{a, b}^{n}\right|\left|\phi^{(b)}(1)\right|+\sum_{k, \ell}\left|d_{k, \ell}^{n}\right|\left|x^{k} \phi^{(\ell)}(x)\right|+\sum_{u, v}\left|e_{u, v}^{n}\right| 2 \max _{|t| \leq 1}\left|t^{n} \phi^{(v)}(t)\right| \\
& \leq \sum_{i, j}\left|c_{a, b}^{n}\right|\left|\phi^{(b)}(1)\right|+\sum_{k, \ell}\left|d_{k, \ell}^{n}\right||x|^{\sigma}\left|\phi^{(\ell)}(x)\right|+\sum_{u, v}\left|e_{u, v}^{n}\right| 2 \max _{|t| \leq 1}|t|^{\sigma}\left|\phi^{(v)}(t)\right| .
\end{aligned}
$$

Let $m, i, j \in \mathbb{N}$ with $i+j \leq m$. By applying the above inequality to the function $x^{i} \phi^{(j)}$, and expressing each derivative $\left(x^{i} \phi^{(j)}\right)^{(r)}$ as a linear combination of functions of the form $x^{p} \phi^{(q)}$ with $p+q \leq i+j+r$, it follows that there is some $C \geq 1$, depending only on $\sigma$ and $m$, such that

$$
\left|x^{i} \phi^{(j)}(x)\right| \leq C\|\phi\|_{\mathcal{S}_{w, \sigma}^{i+j+M_{n}}}
$$

for $|x| \leq 1$. By (18) and (19), $\|\phi\|_{\mathcal{S}^{m}} \leq C\|\phi\|_{\mathcal{S}_{w, \sigma}^{m_{\sigma}}}$ because $m_{\sigma}=m+M_{n}$.

Now, let us prove Claim 1. By induction on $n$ and using integration by parts, it is easy to prove that

$$
\int_{x}^{1} t^{n} \phi^{(n+1)}(t) \mathrm{d} t=\sum_{r=0}^{n}(-1)^{n-r} \frac{n !}{r !}\left(\phi^{(r)}(1)-x^{r} \phi^{(r)}(x)\right) .
$$

This shows directly Claim 1 for $n \in\{0,1\}$. Proceeding by induction, let $n \geq 2$ and assume that Claim 1 holds for $n-1$. By (20), it is enough to find appropriate expressions of $x^{r} \phi^{(r)}(x)$ for $0<r<n$. For that purpose, apply Claim 1 for $n-1$ to each function $\phi^{(r)}$, and multiply the resulting equality by $x^{r}$ to get

$$
\begin{aligned}
x^{r} \phi^{(r)}(x)= & \sum_{a, b} c_{a, b}^{n-1} x^{r+a} \phi^{(r+b)}(1)+\sum_{k, \ell} d_{k, \ell}^{n-1} x^{r+k} \phi^{(r+\ell)}(x) \\
& +\sum_{u, v} e_{u, v}^{n-1} x^{r+u} \int_{x}^{1} t^{n-1} \phi^{(r+v)}(t) \mathrm{d} t
\end{aligned}
$$

where $a, b, k, \ell, u$ and $v$ run in finite subsets of $\mathbb{N}$ with $b, \ell, v \leq M_{n-1}$ and $k \geq n-1$; thus $r+k \geq n$ and $r+b, r+\ell, r+v \leq n-1+M_{n-1}=M_{n}-1$. Therefore it only remains to rise the exponent of $t$ by a unit in the integrals of the last sum. Once more, integration by parts makes the job:

$$
\int_{x}^{1} t^{n} \phi^{(r+v+1)}(t) \mathrm{d} t=\phi^{(r+v)}(1)-x^{n} \phi^{(r+v)}(x)-n \int_{x}^{1} t^{n-1} \phi^{(r+v)} \mathrm{d} t .
$$

Lemma 3.3. If $\sigma<0$, then $\mathcal{S}^{m+1} \subset \mathcal{S}_{w, \sigma}^{m}$ continuously.

Proof. This is proved by induction on $m$. For $\phi \in C_{\mathrm{ev}}^{\infty}$ and $|x| \geq 1$, we have $|x|^{\sigma}|\phi(x)| \leq|\phi(x)|$, obtaining \|\|$_{\mathcal{S}_{w, \sigma}^{0}} \leq\|\|_{\mathcal{S}^{0}}$ on $C_{\mathrm{ev}}^{\infty}$. On the other hand, for $\phi \in C_{\mathrm{odd}}^{\infty}$ and $\psi=x^{-1} \phi \in C_{\mathrm{ev}}^{\infty}$, we get

$$
|x|^{\sigma}|\phi(x)| \leq \begin{cases}|\psi(x)| & \text { if } 0<|x| \leq 1 \\ |\phi(x)| & \text { if }|x| \geq 1\end{cases}
$$

So $\|\phi\|_{\mathcal{S}_{w, \sigma}^{0}} \leq \max \left\{\|\phi\|_{\mathcal{S}^{0}},\|\psi\|_{\mathcal{S}^{0}}\right\} \leq\|\phi\|_{\mathcal{S}^{1}}$ by $(1)$. 
Now, assume that $m \geq 1$ and the result holds for $m-1$. Let $i, j \in \mathbb{N}$ such that $i+j \leq m$, and let $\phi \in \mathcal{S}_{\text {ev }} \cup \mathcal{S}_{\text {odd }}$. Independently of the parity of $\phi$ and $i+j$, we have $|x|^{\sigma}\left|x^{i} \phi^{(j)}(x)\right| \leq\left|x^{i} \phi^{(j)}(x)\right|$ for $|x| \geq 1$.

Suppose that $\phi \in \mathcal{S}_{\text {ev }}$. If $i=0$ and $j$ is odd, then $\phi^{(j)} \in \mathcal{S}_{\text {odd }}$. Thus there is some $\psi \in \mathcal{S}_{\text {ev }}$ such that $\phi^{(j)}=x \psi$, obtaining $|x|^{\sigma}\left|\phi^{(j)}(x)\right| \leq|\psi(x)|$ for $0<|x| \leq 1$. If $i+j$ is odd and $i>0$, then $|x|^{\sigma}\left|x^{i} \phi^{(j)}(x)\right| \leq\left|x^{i-1} \phi^{(j)}(x)\right|$ for $0<|x| \leq 1$. Hence, by (1), there is some $C>0$, independent of $\phi$, such that

$$
\|\phi\|_{\mathcal{S}_{w, \sigma}^{m}} \leq C \max \left\{\|\phi\|_{\mathcal{S}^{m}},\|\psi\|_{\mathcal{S}^{0}}\right\} \leq C \max \left\{\|\phi\|_{\mathcal{S}^{m}},\left\|\phi^{(j)}\right\|_{\mathcal{S}^{1}}\right\} \leq C\|\phi\|_{\mathcal{S}^{m+1}} .
$$

Finally, assume $\phi \in \mathcal{S}_{\text {odd }}$, and let $\psi=x^{-1} \phi \in \mathcal{S}_{\text {ev }}$. If $i$ is even and $j=0$, then $|x|^{\sigma}\left|x^{i} \phi(x)\right| \leq$ $\left|x^{i} \psi(x)\right|$ for $0<|x| \leq 1$. If $i+j$ is even and $j>0$, then

$$
|x|^{\sigma}\left|x^{i} \phi^{(j)}(x)\right| \leq\left|x^{i} \psi^{(j)}(x)\right|+j|x|^{\sigma}\left|x^{i} \psi^{(j-1)}(x)\right|
$$

for $0<|x| \leq 1$ because $\left[\frac{\mathrm{d}^{j}}{\mathrm{~d} x^{j}}, x\right]=j \frac{\mathrm{d}^{j-1}}{\mathrm{~d} x^{j-1}}$. Therefore, by (1) and the induction hypothesis, there are some $C^{\prime}, C^{\prime \prime}>0$, independent of $\phi$, such that

$$
\|\phi\|_{\mathcal{S}_{w, \sigma}^{m}} \leq C^{\prime} \max \left\{\|\phi\|_{\mathcal{S}^{m}},\|\psi\|_{\mathcal{S}^{m}}+\|\psi\|_{\mathcal{S}_{w, \sigma}^{m-1}}\right\} \leq C^{\prime \prime}\|\phi\|_{\mathcal{S}^{m+1}}
$$

Lemma 3.4. If $\sigma<0$, then $\mathcal{S}_{w, \sigma}^{m+1} \subset \mathcal{S}^{m}$ continuously.

Proof. Let $i, j \in \mathbb{N}$ such that $i+j \leq m$. Since

$$
\left|x^{i} \phi^{(j)}(x)\right| \leq \begin{cases}|x|^{\sigma}\left|x^{i} \phi^{(j)}(x)\right| & \text { if } 0<|x| \leq 1 \\ |x|^{\sigma}\left|x^{i+1} \phi^{(j)}(x)\right| & \text { if }|x| \geq 1\end{cases}
$$

for any $\phi \in C^{\infty}$, we get $\|\phi\|_{\mathcal{S}^{m}} \leq\|\phi\|_{\mathcal{S}_{w, \sigma}^{m+1}}$.

Corollary 3.5. $\mathcal{S}=\mathcal{S}_{w, \sigma}$ as Fréchet spaces.

Corollary 3.6. $x^{-1}$ defines a bounded operator $\mathcal{S}_{w, \sigma, \text { odd }}^{m^{\prime}} \rightarrow \mathcal{S}_{w, \sigma, \mathrm{ev}}^{m}$, where

$$
m^{\prime}= \begin{cases}m+2+\frac{1}{2}\lceil\sigma\rceil(\lceil\sigma\rceil+3) & \text { if } \sigma \geq 0 \\ m+3 & \text { if } \sigma<0 .\end{cases}
$$

Proof. If $\sigma \geq 0$, the composite

$$
\mathcal{S}_{w, \sigma, \text { odd }}^{m+2+\frac{1}{2}\lceil\sigma\rceil(\lceil\sigma\rceil+3)} \hookrightarrow \mathcal{S}_{\text {odd }}^{m+\lceil\sigma\rceil+1} \stackrel{x^{-1}}{\longrightarrow} \mathcal{S}_{\mathrm{ev}}^{m+\lceil\sigma\rceil} \hookrightarrow \mathcal{S}_{w, \sigma, \mathrm{ev}}^{m}
$$

is bounded by Lemmas 3.1 and 3.2. If $\sigma<0$, the composite

$$
\mathcal{S}_{w, \sigma, \text { odd }}^{m+3} \hookrightarrow \mathcal{S}_{\text {odd }}^{m+2} \stackrel{x^{-1}}{\longrightarrow} \mathcal{S}_{\mathrm{ev}}^{m+1} \hookrightarrow \mathcal{S}_{w, \sigma, \mathrm{ev}}^{m},
$$

is bounded by Lemmas 3.3 and 3.4.

Corollary 3.7. $x^{-1}$ defines a continuous operator $\mathcal{S}_{w, \sigma, \text { odd }} \rightarrow \mathcal{S}_{w, \sigma, \mathrm{ev}}$.

Lemma 3.8. $\mathcal{S}_{w, \sigma, \text { ev } / \text { odd }}^{M_{m, \text { evd }}} \subset \mathcal{S}_{\sigma \text {,ev } / \text { odd }}^{m}$ continuously, where

$$
M_{m, \mathrm{ev} / \mathrm{odd}}= \begin{cases}\frac{3 m}{2}+\frac{m}{4}\lceil\sigma\rceil(\lceil\sigma\rceil+3) & \text { if } \sigma \geq 0 \text { and } m \text { is even, } \\ 2 m & \text { if } \sigma<0 \text { and } m \text { is even, }\end{cases}
$$




$$
\begin{aligned}
& M_{m, \mathrm{ev}}= \begin{cases}\frac{3 m-1}{2}+\frac{m-1}{4}\lceil\sigma\rceil(\lceil\sigma\rceil+3) & \text { if } \sigma \geq 0 \text { and } m \text { is odd, } \\
2 m-1 & \text { if } \sigma<0 \text { and } m \text { is odd, }\end{cases} \\
& M_{m, \text { odd }}= \begin{cases}\frac{3 m+1}{2}+\frac{m+1}{4}\lceil\sigma\rceil(\lceil\sigma\rceil+3) & \text { if } \sigma \geq 0 \text { and } m \text { is odd, } \\
2 m+1 & \text { if } \sigma<0 \text { and } m \text { is odd. }\end{cases}
\end{aligned}
$$

Proof. This follows by induction on $m$. It is true for $m=0$ because $\mathcal{S}_{w, \sigma}^{0}=\mathcal{S}_{\sigma}^{0}$ as Banach spaces. Now, let $m \geq 1$, and assume that the result holds for $m-1$.

For $\phi \in C_{\mathrm{ev}}^{\infty}, i+j \leq m$ with $j>0$, and $x \in \mathbb{R}$, we have $\left|x^{i}\left(T_{\sigma}^{j} \phi\right)(x)\right|=\left|x^{i}\left(T_{\sigma}^{j-1} \phi^{\prime}\right)(x)\right|$ with $\phi^{\prime} \in C_{\text {odd }}^{\infty}$, obtaining $\|\phi\|_{\mathcal{S}_{\sigma}^{m}} \leq\left\|\phi^{\prime}\right\|_{\mathcal{S}_{\sigma}^{m-1}}$. But, by the induction hypothesis and since $M_{m, \mathrm{ev}}=M_{m-1, \text { odd }}+1$, there are some $C, C^{\prime}>0$, independent of $\phi$, such that

$$
\left\|\phi^{\prime}\right\|_{\mathcal{S}_{\sigma}^{m-1}} \leq C\left\|\phi^{\prime}\right\|_{\mathcal{S}_{w, \sigma}^{M_{m-1, \text { odd }}}} \leq C^{\prime}\|\phi\|_{\mathcal{S}_{w, \sigma}^{M_{m, \mathrm{ev}}}}
$$

For $\phi \in C_{\text {odd }}^{\infty}$, let $\psi=x^{-1} \phi$, and take $i, j$ and $x$ as above. Then

$$
\left|x^{i}\left(T_{\sigma}^{j} \phi\right)(x)\right| \leq\left|x^{i}\left(T_{\sigma}^{j-1} \phi^{\prime}\right)(x)\right|+2|\sigma|\left|x^{i}\left(T_{\sigma}^{j-1} \psi\right)(x)\right|
$$

with $\phi^{\prime}, \psi \in C_{\mathrm{ev}}^{\infty}$, obtaining $\|\phi\|_{\mathcal{S}_{\sigma}^{m}} \leq\left\|\phi^{\prime}\right\|_{\mathcal{S}_{\sigma}^{m-1}}+2|\sigma|\|\psi\|_{\mathcal{S}_{\sigma}^{m-1}}$. But, by the induction hypothesis, Corollary 3.6, and since

$$
M_{m, \mathrm{odd}}= \begin{cases}M_{m-1, \mathrm{ev}}+2+\frac{1}{2}\lceil\sigma\rceil(\lceil\sigma\rceil+3) & \text { if } \sigma \geq 0, \\ M_{m-1, \mathrm{ev}}+3 & \text { if } \sigma<0,\end{cases}
$$

there are some $C, C^{\prime}>0$, independent of $\phi$, such that

$$
\left\|\phi^{\prime}\right\|_{\mathcal{S}_{\sigma}^{m-1}}+2|\sigma|\|\psi\|_{\mathcal{S}_{\sigma}^{m-1}} \leq C\left(\left\|\phi^{\prime}\right\|_{\mathcal{S}_{w, \sigma}^{M_{m-1, \mathrm{ev}}}}+\|\psi\|_{\mathcal{S}_{w, \sigma}^{M_{m-1, \mathrm{ev}}}}\right) \leq C^{\prime}\|\phi\|_{\mathcal{S}_{w, \sigma}^{M_{m, \mathrm{odd}}}}
$$

Corollary 3.9. $\mathcal{S}_{w, \sigma} \subset \mathcal{S}_{\sigma}$ continuously.

Corollary 3.10. $\mathcal{S}_{\mathrm{ev} / \text { odd }}^{M_{m, \mathrm{edd}}^{\prime}} \subset \mathcal{S}_{\sigma \text {,ev/odd }}^{m}$ continuously, where, with the notation of Lemma 3.8,

$$
M_{m, \mathrm{ev} / \mathrm{odd}}^{\prime}= \begin{cases}M_{m, \mathrm{ev} / \mathrm{odd}}+\lceil\sigma\rceil & \text { if } \sigma \geq 0, \\ M_{m, \mathrm{ev} / \mathrm{odd}}+1 & \text { if } \sigma<0 .\end{cases}
$$

Proof. This follows from Lemmas 3.1, 3.3 and 3.8.

\section{Perturbed Sobolev spaces}

Observe that $\mathcal{S}_{\sigma} \subset L^{2}\left(\mathbb{R},|x|^{2 \sigma} \mathrm{d} x\right)$. Like in the case where $\mathcal{S}$ is considered as domain, it is easy to check that, in $L^{2}\left(\mathbb{R},|x|^{2 \sigma} \mathrm{d} x\right)$, with domain $\mathcal{S}_{\sigma}, B$ is adjoint of $B^{\prime}$ and $L$ is symmetric.

Lemma 4.1. $\mathcal{S}_{\sigma}$ is a core ${ }^{4}$ of $\mathcal{L}$.

Proof. Let $R$ denote the restriction of $\mathcal{L}$ to $\mathcal{S}_{\sigma}$. Then $\mathcal{L} \subset \bar{R} \subset R^{*} \subset \mathcal{L}^{*}=\mathcal{L}$ in $L^{2}\left(\mathbb{R},|x|^{2 \sigma} \mathrm{d} x\right)$ because $\mathcal{S} \subset \mathcal{S}_{\sigma}$ by Corollaries 3.5 and 3.9.

\footnotetext{
${ }^{4}$ Recall that a core of a closed densely defined operator $T$ between Hilbert spaces is any subspace of its domain $\mathcal{D}(T)$ which is dense with the graph norm.
} 
For each $m \in \mathbb{R}$, let $W_{\sigma}^{m}$ be the Hilbert space completion of $\mathcal{S}$ with respect to the scalar product $\langle,\rangle_{W_{\sigma}^{m}}$ defined by $\langle\phi, \psi\rangle_{W_{\sigma}^{m}}=\left\langle(1+\mathcal{L})^{m} \phi, \psi\right\rangle_{\sigma}$. The corresponding norm will be denoted by \|\|$_{W_{\sigma}^{m}}$, whose equivalence class is independent of the parameter $s$ used to define $L$. In particular, $W_{\sigma}^{0}=L^{2}\left(\mathbb{R},|x|^{2 \sigma} \mathrm{d} x\right)$. As usual, $W_{\sigma}^{m^{\prime}} \subset W_{\sigma}^{m}$ when $m^{\prime}>m$, and let $W_{\sigma}^{\infty}=$ $\bigcap_{m} W_{\sigma}^{m}$ with the induced Fréchet topology. Once more, there are direct sum decompositions into subspaces of even and odd (generalized) functions, $W_{\sigma}^{m}=W_{\sigma \text {,ev }}^{m} \oplus W_{\sigma, \text { odd }}^{m}(m \in[0, \infty])$. By Lemma $4.1, \mathcal{S}_{\sigma}$ can be used instead of $\mathcal{S}$ in the definition of $W_{\sigma}^{m}$.

Obviously, $L$ defines a bounded operator $W_{\sigma}^{m+2} \rightarrow W_{\sigma}^{m}$ for each $m$, and therefore a continuous operator on $W_{\sigma}^{\infty}$. By (6), $\Sigma$ defines a bounded operator on each $W_{\sigma}^{m}$, and therefore a continuous operator on $W_{\sigma}^{\infty}$. Moreover $B$ and $B^{\prime}$ define bounded operators $W_{\sigma}^{m+1} \rightarrow W_{\sigma}^{m}$ for each $m \in \mathbb{N}$; this follows easily by induction on $m$, using (3) and (4) (the details of the proof are omitted because this observation will not be used). Thus $L, \Sigma, B$ and $B^{\prime}$ define bounded operators on $W_{\sigma}^{\infty}$. Also on the spaces $W_{\sigma}^{m}$, the parity of (generalized) functions is preserved by $L$ and $\Sigma$, and reversed by $B$ and $B^{\prime}$. Observe that $B^{\prime}$ is not adjoint of $B$ in $W_{\sigma}^{m}$ for $m \neq 0$.

The motivation of our tour through perturbed Schwartz spaces is the following embedding results; the second one is a version of the Sobolev embedding theorem.

Proposition 4.2. $\mathcal{S}_{\sigma}^{m+1} \subset W_{\sigma}^{m}$ continuously for all $m \in \mathbb{N}$.

Proposition 4.3. For all $m \in \mathbb{N}, W_{\sigma}^{m^{\prime}} \subset \mathcal{S}_{\sigma}^{m}$ continuously if $m^{\prime}-m>1$.

Corollary 4.4. $\mathcal{S}_{\sigma}=W_{\sigma}^{\infty}$ as Fréchet spaces.

For each non-commutative polynomial $p$ (of two variables, $X$ and $Y$ ), let $p^{\prime}$ denote the noncommutative polynomial obtained by reversing the order of the variables in $p$; e.g., if $p(X, Y)=$ $X^{2} Y^{3} X$, then $p^{\prime}(X, Y)=X Y^{3} X^{2}$. It will be said that $p$ is symmetric if $p(X, Y)=p^{\prime}(Y, X)$. Note that $p^{\prime}(Y, X) p(X, Y)$ is symmetric for any $p$. Given any non-commutative polynomial $p$, the continuous operators $p\left(B, B^{\prime}\right)$ and $p^{\prime}\left(B^{\prime}, B\right)$ on $\mathcal{S}_{\sigma}$ are adjoint of each other in $L^{2}\left(\mathbb{R},|x|^{2 \sigma} \mathrm{d} x\right)$; thus $p\left(B, B^{\prime}\right)$ is a symmetric operator if $p$ is symmetric.

Lemma 4.5. For $m \in \mathbb{N}$, we have $(1+L)^{m}=\sum_{a} q_{a}^{\prime}\left(B^{\prime}, B\right) q_{a}\left(B, B^{\prime}\right)$ for some finite family of homogeneous non-commutative polynomials $q_{a}$ of degree $\leq m$.

Proof. The result follows easily from the following assertions.

Claim 2. If $m$ is even, then $L^{m}=g_{m}\left(B, B^{\prime}\right)^{2}$ for some symmetric homogeneous non-commutative polynomial $g_{m}$ of degree $m$.

Claim 3. If $m$ is odd, then

$$
L^{m}=g_{m, 1}^{\prime}\left(B^{\prime}, B\right) g_{m, 1}\left(B, B^{\prime}\right)+g_{m, 2}^{\prime}\left(B^{\prime}, B\right) g_{m, 2}\left(B, B^{\prime}\right)
$$

for some homogeneous non-commutative polynomials $g_{m, 1}$ and $g_{m, 2}$ of degree $m$.

If $m$ is even, then $L^{m / 2}=g_{m}\left(B, B^{\prime}\right)$ for some symmetric homogeneous non-commutative polynomial $g_{m}$ of degree $m$ by (3). So $L^{m}=g_{m}\left(B, B^{\prime}\right)^{2}$, showing Claim 2 .

If $m$ is odd, write $L^{\lfloor m / 2\rfloor}=f_{m}\left(B, B^{\prime}\right)$ as above for some symmetric homogeneous noncommutative polynomial $f_{m}$ of degree $m-1$. Then, by (3),

$$
L^{m}=\frac{1}{2} f_{m}\left(B, B^{\prime}\right)\left(B B^{\prime}+B^{\prime} B\right) f_{m}\left(B, B^{\prime}\right) .
$$

Thus Claim 3 follows with

$$
g_{m, 1}\left(B, B^{\prime}\right)=\frac{1}{\sqrt{2}} B^{\prime} f_{m}\left(B, B^{\prime}\right), \quad g_{m, 2}\left(B, B^{\prime}\right)=\frac{1}{\sqrt{2}} B f_{m}\left(B, B^{\prime}\right) .
$$


Proof of Proposition 4.2. By the definitions of $B$ and $B^{\prime}$, and (15)-(17), for each homogeneous non-commutative polynomial $p$ of three variables with degree $d \leq m+1$, there exists some $C_{p}>0$ such that, for all $\phi \in \mathcal{S}_{\sigma}$ : if $\sigma<0,|x| \leq 1$, and $\phi$ and $d$ have the same parity, then $\left|\left(p\left(x, B, B^{\prime}\right) \phi\right)(x)\right| \leq C_{p}\|\phi\|_{\mathcal{S}_{\sigma}^{m+1}}$; and, otherwise, $|x|^{\sigma}\left|\left(p\left(x, B, B^{\prime}\right) \phi\right)(x)\right| \leq C_{p}\|\phi\|_{\mathcal{S}_{\sigma}^{m+1}}$.

With the notation of Lemma 4.5 , let $d_{a}$ denote the degree of each $q_{a}$, and let $\bar{q}_{a}\left(x, B, B^{\prime}\right)=$ $x q_{a}\left(B, B^{\prime}\right)$. If $\sigma \geq 0$, then

$$
\begin{aligned}
\|\phi\|_{W_{\sigma}^{m}}^{2}= & \sum_{a}\left\|q_{a}\left(B, B^{\prime}\right) \phi\right\|_{\sigma}^{2}=\sum_{a} \int_{-\infty}^{\infty}\left|\left(q_{a}\left(B, B^{\prime}\right) \phi\right)(x)\right|^{2}|x|^{2 \sigma} \mathrm{d} x \\
& \leq 2 \sum_{a}\left(C_{q_{a}}^{2}+C_{\bar{q}_{a}}^{2} \int_{1}^{\infty} x^{-2} \mathrm{~d} x\right)\|\phi\|_{\mathcal{S}_{\sigma}^{m+1}}^{2}
\end{aligned}
$$

for $\phi \in \mathcal{S}_{\sigma}$. Similarly, if $\sigma<0$, then $\|\phi\|_{W_{\sigma}^{m}}^{2}$ is bounded by

$$
2\left(\sum_{d_{a} \text { even }} C_{q_{a}}^{2} \int_{0}^{1} x^{2 \sigma} \mathrm{d} x+\sum_{d_{a} \text { odd }} C_{q_{a}}^{2}+\sum_{a} C_{\bar{q}_{a}}^{2} \int_{1}^{\infty} x^{-2} \mathrm{~d} x\right)\|\phi\|_{\mathcal{S}_{\sigma}^{m+1}}^{2}
$$

for $\phi \in \mathcal{S}_{\sigma, \mathrm{ev}}$, and by

$$
2\left(\sum_{d_{a} \text { even }} C_{q_{a}}^{2}+\sum_{d_{a} \text { odd }} C_{q_{a}}^{2} \int_{0}^{1} x^{2 \sigma} \mathrm{d} x+\sum_{a} C_{\bar{q}_{a}}^{2} \int_{1}^{\infty} x^{-2} \mathrm{~d} x\right)\|\phi\|_{\mathcal{S}_{\sigma}^{m+1}}^{2}
$$

for $\phi \in \mathcal{S}_{\sigma, \text { odd }}$.

For $c=\left(c_{k}\right)$ and $c^{\prime}=\left(c_{k}^{\prime}\right)$ in $\mathbb{R}^{\mathbb{N}}$, and $m \in \mathbb{R}$, the expressions $\|c\|_{\mathcal{C}_{m}}=\sup _{k}\left|c_{k}\right|(1+k)^{m}$ and $\left\langle c, c^{\prime}\right\rangle_{\ell_{m}^{2}}=\sum_{k} c_{k} c_{k}^{\prime}(1+k)^{m}$ define a norm and scalar product with possible infinite values, and let \|\|$_{\ell_{m}^{2}}$ denote the norm with possible infinite values induced by $\langle,\rangle_{\ell_{m}^{2}}$. Then $\mathcal{C}_{m}=\left\{c \in \mathbb{R}^{\mathbb{N}} \mid\right.$ $\left.\|c\|_{\mathcal{C}_{m}}<\infty\right\}$ becomes a Banach space with \|\|$_{\mathcal{C}_{m}}$, and $\ell_{m}^{2}=\left\{c \in \mathbb{R}^{\mathbb{N}} \mid\|c\|_{\ell_{m}^{2}}<\infty\right\}$ is a Hilbert space with $\langle,\rangle_{\ell_{m}^{2}}$. If $m^{\prime}>m$, then $\mathcal{C}_{m^{\prime}} \subset \mathcal{C}_{m}$ and $\ell_{m^{\prime}}^{2} \subset \ell_{m}^{2}$ continuously. Let $\mathcal{C}_{\infty}=\bigcap_{m} \mathcal{C}_{m}$ and $\ell_{\infty}^{2}=\bigcap_{m} \ell_{m}^{2}$, with the induced Fréchet topologies. It is said that $c$ is even/odd if $c_{k}=0$ for odd/even $k$. There are direct sum decompositions into subspaces of even and odd sequences, $\mathcal{C}_{m}=\mathcal{C}_{m, \mathrm{ev}} \oplus \mathcal{C}_{m, \text { odd }}$ and $\ell_{m}^{2}=\ell_{m, \mathrm{ev}}^{2} \oplus \ell_{m, \text { odd }}^{2}(m \in \mathbb{R} \cup\{\infty\})$.

Lemma 4.6. $\ell_{2 m}^{2} \subset \mathcal{C}_{m}$ and $\mathcal{C}_{m^{\prime}} \subset \ell_{m}^{2}$ continuously if $2 m^{\prime}-m>1$.

Proof. It is easy to see that $\|c\|_{\mathcal{C}_{m}} \leq\|c\|_{\ell_{2 m}^{2}}$ and $\|c\|_{\ell_{m}^{2}} \leq\|c\|_{\mathcal{C}_{m^{\prime}}}\left(\sum_{k}(1+k)^{m-2 m^{\prime}}\right)^{1 / 2}$ for any $c \in \mathcal{C}_{\infty}$, where the last series is convergent because $m-2 m^{\prime}<-1$.

Corollary 4.7. $\ell_{\infty}^{2}=\mathcal{C}_{\infty}$ as Fréchet spaces.

According to Section 2.2, the "Fourier coefficients" mapping $\phi \mapsto\left(\left\langle\phi_{k}, \phi\right\rangle_{\sigma}\right)$ defines a quasiisometry $W_{\sigma}^{m} \rightarrow \ell_{m}^{2}$ for all finite $m$, and therefore an isomorphism $W_{\sigma}^{\infty} \rightarrow \mathcal{C}_{\infty}$ of Fréchet espaces. This map is compatible with the decompositions into even and odd subspaces.

Corollary 4.8. Any $\phi \in L^{2}\left(\mathbb{R},|x|^{2 \sigma} \mathrm{d} x\right)$ is in $\mathcal{S}_{\sigma}$ if and only if its "Fourier coefficients" $\left\langle\phi_{k}, \phi\right\rangle_{\sigma}$ are rapidly decreasing on $k$.

Proof. By Corollary 4.4, the "Fourier coefficients" mapping defines an isomorphism $\mathcal{S}_{\sigma} \rightarrow \mathcal{C}_{\infty}$ of Fréchet spaces.

The operator $\ell_{m^{\prime}}^{2} \hookrightarrow \ell_{m}^{2}$ is compact if $m^{\prime}>m$ (see e.g. [28, Theorem 5.8]). So, by using the "Fourier coefficients" mapping, the operator $W_{\sigma}^{m^{\prime}} \hookrightarrow W_{\sigma}^{m}$ is compact if $m^{\prime}>m$ (a version of the Rellich theorem). 
Proof of Proposition 4.3. For $\phi \in \mathcal{S}_{\sigma}$, its "Fourier coefficients" $c_{k}=\left\langle\phi_{k}, \phi\right\rangle_{\sigma}$ form a sequence $c=\left(c_{k}\right)$ in $\mathcal{C}_{\infty}$, and $\sum_{k}\left|c_{k}\right|(1+k)^{m / 2} \leq\|c\|_{\ell_{m^{\prime}}}\left(\sum_{k}(1+k)^{m-m^{\prime}}\right)^{1 / 2}$ by Cauchy-Schwartz inequality, where the last series is convergent since $m-m^{\prime}<-1$. Therefore there is some $C>0$, independent of $\phi$, such that

$$
\sum_{k}\left|c_{k}\right|(1+k)^{m / 2} \leq C\|\phi\|_{W_{\sigma}^{m^{\prime}}}
$$

On the other hand, for all $i, j \in \mathbb{N}$ with $i+j \leq m$, there is some homogeneous noncommutative polynomial $p_{i j}$ of degree $i+j$ such that $x^{i} T_{\sigma}^{j}=p_{i j}\left(B, B^{\prime}\right)$. Then, by (8)-(10), there is some $C_{i j}>0$, independent of $\phi$, such that

$$
\left|\left\langle\phi_{k}, x^{i} T_{\sigma}^{j} \phi\right\rangle_{\sigma}\right| \leq C_{i j}(1+k)^{m / 2} \sum_{|\ell-k| \leq m}\left|c_{\ell}\right| .
$$

Assume that $\sigma \geq 0$. By (21), (22) and Theorem 2.1(i), there is some $C_{i j}^{\prime}>0$, independent of $\phi$ and $x_{0}$, so that

$$
\begin{aligned}
\left|x_{0}\right|^{\sigma}\left|\left(x^{i} T_{\sigma}^{j} \phi\right)\left(x_{0}\right)\right| & \leq\left|x_{0}\right|^{\sigma} \sum_{k}\left|\left\langle\phi_{k}, x^{i} T_{\sigma}^{j} \phi\right\rangle_{\sigma} \| \phi_{k}\left(x_{0}\right)\right| \\
& =\sum_{k}\left|\left\langle\phi_{k}, x^{i} T_{\sigma}^{j} \phi\right\rangle_{\sigma}\right|\left|\xi_{k}\left(x_{0}\right)\right| \leq C_{i j}^{\prime}\|\phi\|_{W_{\sigma}^{m^{\prime}}}
\end{aligned}
$$

for all $x_{0} \in \mathbb{R}$. Hence $\|\phi\|_{\mathcal{S}_{\sigma}^{m}} \leq C^{\prime}\|\phi\|_{W_{\sigma}^{m^{\prime}}}$ for some $C^{\prime}>0$ independent of $\phi$.

Now, suppose that $\sigma<0$. From (21), (22), and Theorem 2.1(ii),(iii), it follows that there is some $C_{i j}^{\prime}>0$, independent of $\phi$ and $x_{0}$, such that

$$
\left|\left(x^{i} T_{\sigma}^{j} \phi\right)\left(x_{0}\right)\right| \leq \sum_{k}\left|\left\langle\phi_{k}, x^{i} T_{\sigma}^{j} \phi\right\rangle_{\sigma}\left\|\phi_{k}\left(x_{0}\right) \mid \leq C_{i j}^{\prime}\right\| \phi \|_{W_{\sigma}^{m^{\prime}}}\right.
$$

if $\phi$ and $i+j$ have the same parity, and $\left|x_{0}\right| \leq 1$; otherwise, $\left|x_{0}\right|^{\sigma}\left|\left(x^{i} T_{\sigma}^{j} \phi\right)\left(x_{0}\right)\right| \leq C_{i j}^{\prime}\|\phi\|_{W_{\sigma}^{m^{\prime}}}$ like in (23). So $\|\phi\|_{\mathcal{S}_{\sigma}^{m}} \leq C^{\prime}\|\phi\|_{W_{\sigma}^{m^{\prime}}}$ with $C^{\prime}>0$ independent of $\phi$.

As suggested by (14), consider the mapping $c=\left(c_{k}\right) \mapsto \Xi(c)=\left(d_{\ell}\right)$, where $c$ is odd and $\Xi(c)$ is even, with

$$
d_{\ell}=\sum_{k \in\{\ell+1, \ell+3, \ldots\}}(-1)^{\frac{k-\ell-1}{2}} \sqrt{\frac{(k-1)(k-3) \cdots(\ell+2) 2 s}{(k+2 \sigma)(k-2+2 \sigma) \cdots(\ell+1+2 \sigma)}} c_{k}
$$

for $\ell$ even, assuming that this series is convergent.

Lemma 4.9. $\Xi$ defines a bounded map $\ell_{m^{\prime}, \text { odd }}^{2} \rightarrow \mathcal{C}_{m, \mathrm{ev}}$ if $m^{\prime}-m>1$.

Proof. By the Cauchy-Schwartz inequality,

$$
\begin{aligned}
\|d\|_{\mathcal{C}_{m}} & =\sup _{\ell} \sum_{k \in\{\ell+1, \ell+3, \ldots\}} \sqrt{\frac{(k-1)(k-3) \cdots(\ell+2) 2 s}{(k+2 \sigma)(k-2+2 \sigma) \cdots(\ell+1+2 \sigma)}}\left|c_{k}\right|(1+\ell)^{m} \\
& \leq \sqrt{2 s} \sup _{\ell} \sum_{k \in\{\ell+1, \ell+3, \ldots\}}\left|c_{k}\right|(1+\ell)^{m} \\
& \leq \sqrt{2 s}\|c\|_{\ell_{m^{\prime}}^{2}} \sup _{\ell}\left(\sum_{k \in\{\ell+1, \ell+3, \ldots\}}(1+k)^{-m^{\prime}}(1+\ell)^{m}\right)^{1 / 2}
\end{aligned}
$$




$$
\leq \sqrt{2 s}\|c\|_{\ell_{m^{\prime}}^{2}}\left(\sum_{k}(1+k)^{m-m^{\prime}}\right)^{1 / 2}
$$

where the last series is convergent because $m-m^{\prime}<-1$.

Corollary 4.10. $x^{-1}$ defines a bounded operator $\mathcal{S}_{\sigma, \mathrm{odd}}^{m^{\prime}} \rightarrow \mathcal{S}_{\sigma, \mathrm{ev}}^{m}$ if $2 m^{\prime}>m+6$.

Proof. Since $2 m^{\prime}>m+6$, there are $m_{1}, m_{2} \in \mathbb{R}$ so that $m^{\prime}-m_{2}>2,2 m_{2}-m_{1}>1$ and $m_{1}-m>1$. Then, by Propositions 4.2 and 4.3, Lemmas 4.6 and 4.9, and using the "Fourier coefficients" mapping, we get the composition of bounded maps,

$$
\mathcal{S}_{\sigma, \text { odd }}^{m^{\prime}} \hookrightarrow W_{\sigma, \text { odd }}^{m^{\prime}-1} \rightarrow \ell_{m^{\prime}-1, \text { odd }}^{2} \stackrel{\Xi}{\longrightarrow} \mathcal{C}_{m_{2}, \text { ev }} \hookrightarrow \ell_{m_{1}, \text { ev }}^{2} \rightarrow W_{\sigma, \text { ev }}^{m_{1}} \hookrightarrow \mathcal{S}_{\sigma, \text { ev }}^{m},
$$

which extends $x^{-1}: \mathcal{S}_{\text {odd }} \rightarrow \mathcal{S}_{\text {ev }}$ by (14).

Question 4.11. Is it possible to prove Corollary 4.10 without using (14)?

Corollary 4.12. $x^{-1}$ defines a continuous operator $\mathcal{S}_{\sigma, \mathrm{odd}} \rightarrow \mathcal{S}_{\sigma, \mathrm{ev}}$.

Lemma 4.13. $\mathcal{S}_{\sigma, \mathrm{ev} / \mathrm{odd}}^{M_{m, \mathrm{ev} / \mathrm{odd}}} \subset \mathcal{S}_{w, \sigma, \mathrm{ev} / \mathrm{odd}}^{m}$ continuously for all $m$, where $M_{0, \mathrm{ev} / \mathrm{odd}}=0, M_{1, \mathrm{ev}}=1$, $M_{1, \text { odd }}=M_{2, \text { odd }}=4, M_{2, \text { ev }}=M_{3, \mathrm{ev}}=5, M_{3, \text { odd }}=6$, and $M_{m, \text { ev } / \text { odd }}=m+2$ for $m \geq 4$.

Proof. We proceed by induction on $m$. The case $m=0$ was already indicated in the proof of Lemma 3.8. Now, let $m \geq 1$ and assume that the result holds for $m-1$.

For $\phi \in C_{\mathrm{ev}}^{\infty}, i+j \leq m$ with $j>0$ and $x \in \mathbb{R}$, we have $\left|x^{i} \phi^{(j)}(x)\right|=\left|x^{i}\left(T_{\sigma} \phi\right)^{(j-1)}(x)\right|$ with $T_{\sigma} \phi \in C_{\text {odd }}^{\infty}$, obtaining $\|\phi\|_{\mathcal{S}_{w, \sigma}^{m}} \leq\left\|T_{\sigma} \phi\right\|_{\mathcal{S}_{w, \sigma}^{m-1}}$. But, by the induction hypothesis and because $M_{m, \mathrm{ev}}=M_{m-1, \mathrm{odd}}+1$, there are some $C, C^{\prime}>0$, independent of $\phi$, such that $\left\|T_{\sigma} \phi\right\|_{\mathcal{S}_{w, \sigma}^{m-1}} \leq$ $C\left\|T_{\sigma} \phi\right\|_{\mathcal{S}_{\sigma}^{M_{m-1, \text { odd }}}} \leq C^{\prime}\|\phi\|_{\mathcal{S}_{\sigma}^{M_{m, \mathrm{ev}}}}$.

For $\phi \in C_{\text {odd }}^{\infty}$, let $\psi=x^{-1} \phi$, and take $i, j$ and $x$ as above. We have

$$
\left|x^{i} \phi^{(j)}(x)\right| \leq\left|x^{i}\left(T_{\sigma} \phi\right)^{(j-1)}(x)\right|+2|\sigma|\left|x^{i} \psi^{(j-1)}(x)\right|
$$

with $T_{\sigma} \phi, \psi \in C_{\mathrm{ev}}^{\infty}$, obtaining $\|\phi\|_{\mathcal{S}_{w, \sigma}^{m}} \leq\left\|T_{\sigma} \phi\right\|_{\mathcal{S}_{w, \sigma}^{m-1}}+2|\sigma|\|\psi\|_{\mathcal{S}_{w, \sigma}^{m-1}}$. But, by the induction hypothesis, Corollary 4.10 , and since $M_{m \text {,odd }} \geq M_{m-1, \mathrm{ev}}+1$ and $2 M_{m \text {,odd }}>M_{m-1, \mathrm{ev}}+6$, there are some $C, C^{\prime}>0$, independent of $\phi$, such that

$$
\left\|T_{\sigma} \phi\right\|_{\mathcal{S}_{w, \sigma}^{m-1}}+2|\sigma|\|\psi\|_{\mathcal{S}_{w, \sigma}^{m-1}} \leq C\left(\left\|\phi^{\prime}\right\|_{\mathcal{S}_{\sigma}^{M_{m-1, \mathrm{ev}}}}+\|\psi\|_{\mathcal{S}_{\sigma}^{M_{m-1, \mathrm{ev}}}}\right) \leq C^{\prime}\|\phi\|_{\mathcal{S}_{\sigma}^{M_{m, \mathrm{odd}}}} .
$$

Corollary 4.14. $\mathcal{S}_{\sigma, \mathrm{ev} / \mathrm{odd}}^{M_{m, \mathrm{ev} / \mathrm{dd}}^{\prime}} \subset \mathcal{S}_{\mathrm{ev} / \mathrm{odd}}^{m}$ continuously, where, with the notation of Lemma 4.13, $M_{m, \text { ev } / \text { odd }}^{\prime}=M_{m_{\sigma}, \text { ev } / \text { odd }}$ for $m_{\sigma}=m+1+\frac{1}{2}\lceil\sigma\rceil(\lceil\sigma\rceil+1)$.

Proof. This follows from Lemmas 3.2, 3.4 and 4.13.

Corollary 4.15. $\mathcal{S}_{\sigma}=\mathcal{S}$ as Fréchet spaces.

Proof. This is a consequence of Corollaries 3.10 and 4.14

Corollaries 3.10 and 4.14 and Propositions 4.2 and 4.3 give Theorems 1.1 and 1.2. 


\section{$5 \quad$ Perturbations of $\boldsymbol{H}$ on $\mathbb{R}_{+}$}

Since the function $|x|^{2 \sigma}$ is even, the decomposition $\mathcal{S}=\mathcal{S}_{\text {ev }} \oplus \mathcal{S}_{\text {odd }}$ extends to an orthogonal decomposition

$$
L^{2}\left(\mathbb{R},|x|^{2 \sigma} \mathrm{d} x\right)=L_{\text {ev }}^{2}\left(\mathbb{R},|x|^{2 \sigma} \mathrm{d} x\right) \oplus L_{\text {odd }}^{2}\left(\mathbb{R},|x|^{2 \sigma} \mathrm{d} x\right) .
$$

Let $L_{\text {ev/odd }}$ and $\mathcal{L}_{\text {ev/odd }}$, or $L_{\sigma \text {,ev/odd }}$ and $\mathcal{L}_{\sigma \text {,ev/odd }}$, denote the corresponding components of $L$ and $\mathcal{L} . \quad L_{\text {ev/odd }}$ is essentially self-adjoint in $L_{\text {ev } / \text { odd }}^{2}\left(\mathbb{R},|x|^{2 \sigma} \mathrm{d} x\right)$, and its self-adjoint extension is $\mathcal{L}_{\text {ev/odd }}$, which satisfies an obvious version of Corollary 1.3.

Fix an open subset $U \subset \mathbb{R}_{+}$of full Lebesgue measure. Let $\mathcal{S}_{\text {ev/odd, } U} \subset C^{\infty}(U)$ be the linear subspace of restrictions to $U$ of the functions in $\mathcal{S}_{\text {ev/odd }}$. The restriction to $U$ defines a linear isomorphism $\mathcal{S}_{\text {ev/odd }} \cong \mathcal{S}_{\text {ev/odd }, U}$, and a unitary isomorphism $L_{\text {ev /odd }}^{2}\left(\mathbb{R},|x|^{2 \sigma} \mathrm{d} x\right) \cong L^{2}\left(U, 2 x^{2 \sigma} \mathrm{d} x\right)$. Via these isomorphisms, $L_{\text {ev/odd }}$ corresponds to an operator $L_{\text {ev/odd, } U}$ on $\mathcal{S}_{\text {ev/odd, } U}$, and $\mathcal{L}_{\text {ev/odd }}$ corresponds to a self-adjoint operator $\mathcal{L}_{\text {ev/odd }, U}$ in $L^{2}\left(U, x^{2 \sigma} \mathrm{d} x\right)$; the more explicit notation $L_{\sigma, \mathrm{ev} / \mathrm{odd}, U}$ and $\mathcal{L}_{\sigma, \mathrm{ev} / \mathrm{odd}, U}$ may be used. Let $\phi_{k, U}=\left.\phi_{k}\right|_{U}$, whose norm in $L^{2}\left(U, x^{2 \sigma} \mathrm{d} x\right)$ is $1 / \sqrt{2}$.

Going one step further, for any positive function $h \in C^{2}(U)$, the multiplication by $h$ defines a unitary isomorphism $h: L^{2}\left(U, x^{2 \sigma} \mathrm{d} x\right) \rightarrow L^{2}\left(U, x^{2 \sigma} h^{-2} \mathrm{~d} x\right)$. Thus $h L_{\mathrm{ev}, U} h^{-1}$, with domain $h \mathcal{S}_{\text {ev }, U}$, is essentially self-adjoint in $L^{2}\left(U, x^{2 \sigma} h^{-2} \mathrm{~d} x\right)$, and its self-adjoint extension is $h \mathcal{L}_{\mathrm{ev},+} h^{-1}$. Via these unitary isomorphisms, we get an obvious version of Corollary 1.3 for $h \mathcal{L}_{\mathrm{ev},+} h^{-1}$. By using

$$
\left[\frac{\mathrm{d}}{\mathrm{d} x}, h\right]=h^{\prime}, \quad\left[\frac{\mathrm{d}^{2}}{\mathrm{~d} x^{2}}, h\right]=2 h^{\prime} \frac{\mathrm{d}}{\mathrm{d} x}+h^{\prime \prime}
$$

it easily follows that $h L_{\mathrm{ev}, U} h^{-1}$ has the form of $P$ in Theorem 1.4. Then Theorem 1.4 is a consequence of the following.

Lemma 5.1. For $\sigma>-1 / 2$, a positive function $h \in C^{2}(U)$, and $P=H-2 f_{1} \frac{\mathrm{d}}{\mathrm{d} x}+f_{2}$ with $f_{1} \in C^{1}(U)$ and $f_{2} \in C(U)$, we have $P=h L_{\sigma, \mathrm{ev}, U} h^{-1}$ on $h \mathcal{S}_{\mathrm{ev}, U}$ if and only if $f_{1}, f_{2}$ and $h$ satisfy the conditions of Theorem 1.4.

Proof. By (24),

$$
h^{-1} P h=H-2\left(h^{-1} h^{\prime}+f_{1}\right) \frac{\mathrm{d}}{\mathrm{d} x}-h^{-1} h^{\prime \prime}-2 h^{-1} f_{1} h^{\prime}+f_{2} .
$$

So $P=h L_{\sigma, \mathrm{ev}, U} h^{-1}$ if and only if $h^{-1} h^{\prime}=\sigma x^{-1}-f_{1}$ and $f_{2}=h^{-1} h^{\prime \prime}+2 h^{-1} h^{\prime} f_{1}$, which are easily seen to be equivalent to the conditions of Theorem 1.4.

Remark 5.2. By (24), we get an operator of the same type if $h$ and $\frac{\mathrm{d}}{\mathrm{d} x}$ is interchanged in the operator $P$ of Theorem 1.4.

Remark 5.3. By using (24) with $h=x^{-1}$ on $\mathbb{R}_{+}$, it is easy to check that $L_{\sigma, \text { odd, } \mathbb{R}_{+}}=$ $x L_{1+\sigma, \mathrm{ev}, \mathbb{R}_{+}} x^{-1}$ on $\mathcal{S}_{\text {odd }, \mathbb{R}_{+}}=x \mathcal{S}_{\mathrm{ev}, \mathbb{R}_{+}}$. So no new operators are obtained with this process by using $L_{\sigma, \text { odd }}$ instead of $L_{\sigma \text {,ev }}$.

\section{Acknowledgements}

The first author is partially supported by MICINN and MEC, Grants MTM2008-02640, MTM2011-25656 and PR2009-0409. 


\section{References}

[1] Álvarez López J., Calaza M., Application of the method of Bonan-Clark to the generalized Hermite polynomials, arXiv:1101.5022.

[2] Álvarez López J., Calaza M., Witten's perturbation on strata, arXiv:1205.0348.

[3] Askey R., Wainger S., Mean convergence of expansions in Laguerre and Hermite series, Amer. J. Math. 87 (1965), 695-708.

[4] Bonan S.S., Clark D.S., Estimates of the Hermite and the Freud polynomials, J. Approx. Theory 63 (1990), 210-224.

[5] Brasselet J.P., Hector G., Saralegi M., $\mathcal{L}^{2}$-cohomologie des espaces stratifiés, Manuscripta Math. 76 (1992), 21-32.

[6] Brüning J., Lesch M., Hilbert complexes, J. Funct. Anal. 108 (1992), 88-132.

[7] Cheeger J., On the Hodge theory of Riemannian pseudomanifolds, in Geometry of the Laplace Operator (Proc. Sympos. Pure Math., Univ. Hawaii, Honolulu, Hawaii, 1979), Proc. Sympos. Pure Math., Vol. 36, Amer. Math. Soc., Providence, R.I., 1980, 91-146.

[8] Cheeger J., Spectral geometry of singular Riemannian spaces, J. Differential Geom. 18 (1983), 575-657.

[9] Chihara T.S., Generalized Hermite polynomials, Ph.D. Thesis, Purdue University, 1955.

[10] Chihara T.S., An introduction to orthogonal polynomials, Mathematics and its Applications, Vol. 13, Gordon and Breach Science Publishers, New York, 1978.

[11] Dickinson D., Warsi S.A., On a generalized Hermite polynomial and a problem of Carlitz, Boll. Un. Mat. Ital. 18 (1963), 256-259.

[12] Dunkl C.F., Reflection groups and orthogonal polynomials on the sphere, Math. Z. 197 (1988), 33-60.

[13] Dunkl C.F., Differential-difference operators associated to reflection groups, Trans. Amer. Math. Soc. 311 (1989), 167-183.

[14] Dunkl C.F., Integral kernels with reflection group invariance, Canad. J. Math. 43 (1991), 1213-1227.

[15] Dunkl C.F., Symmetric functions and $B_{N}$-invariant spherical harmonics, J. Phys. A: Math. Gen. 35 (2002), 10391-10408, math.CA/0207122.

[16] Dutta M., Chatterjea S.K., More K.L., On a class of generalized Hermite polynomials, Bull. Inst. Math. Acad. Sinica 3 (1975), 377-381.

[17] Erdélyi A., Asymptotic forms for Laguerre polynomials, J. Indian Math. Soc. 24 (1960), 235-250.

[18] Goresky M., MacPherson R., Morse theory and intersection homology theory, Astérisque 101 (1983), 135192.

[19] Hörmander L., The analysis of linear partial differential operators. I. Distribution theory and Fourier analysis, Grundlehren der Mathematischen Wissenschaften, Vol. 256, 2nd ed., Springer-Verlag, Berlin, 1990.

[20] Mather J., Notes on topological stability, Hardvard University, 1970, available at http://www.math. princeton.edu/facultypapers/mather/notes_on_topological_stability.pdf.

[21] Muckenhoupt B., Asymptotic forms for Laguerre polynomials, Proc. Amer. Math. Soc. 24 (1970), $288-292$.

[22] Muckenhoupt B., Mean convergence of Hermite and Laguerre series. II, Trans. Amer. Math. Soc. 147 (1970), 433-460.

[23] Nagase M., $L^{2}$-cohomology and intersection homology of stratified spaces, Duke Math. J. 50 (1983), 329-368.

[24] Nagase M., Sheaf theoretic $L^{2}$-cohomology, Adv. Stud. Pure Math. 8 (1986), 273-279.

[25] Nowak A., Stempak K., Imaginary powers of the Dunkl harmonic oscillator, SIGMA 5 (2009), 016, 12 pages, arXiv:0902.1958.

[26] Nowak A., Stempak K., Riesz transforms for the Dunkl harmonic oscillator, Math. Z. 262 (2009), 539-556, arXiv:0802.0474.

[27] Plyushchay M., Hidden nonlinear supersymmetries in pure parabosonic systems, Internat. J. Modern Phys. A 15 (2000), 3679-3698, hep-th/9903130.

[28] Roe J., Elliptic operators, topology and asymptotic methods, Pitman Research Notes in Mathematics Series, Vol. 395, 2nd ed., Longman, Harlow, 1998. 
[29] Rosenblum M., Generalized Hermite polynomials and the Bose-like oscillator calculus, in Nonselfadjoint Operators and Related Topics (Beer Sheva, 1992), Oper. Theory Adv. Appl., Vol. 73, Birkhäuser, Basel, 1994, 369-396, math.CA/9307224.

[30] Rösler M., Generalized Hermite polynomials and the heat equation for Dunkl operators, Comm. Math. Phys. 192 (1998), 519-542, q-alg/9703006.

[31] Rösler M., Dunkl operators: theory and applications, in Orthogonal Polynomials and Special Functions (Leuven, 2002), Lecture Notes in Math., Vol. 1817, Springer, Berlin, 2003, 93-135, math.CA/0210366.

[32] Szegö G., Orthogonal polynomials, Colloquium Publications, Vol. 23, 4th ed., Amer. Math. Soc., Providence, R.I., 1975.

[33] Thom R., Ensembles et morphismes stratifiés, Bull. Amer. Math. Soc. 75 (1969), 240-284.

[34] Verona A., Stratified mappings - structure and triangulability, Lecture Notes in Math., Vol. 1102, SpringerVerlag, Berlin, 1984.

[35] Watanabe S., An embedding theorem of Sobolev type for an operator with singularity, Proc. Amer. Math. Soc. 125 (1997), 839-848.

[36] Watanabe S., A generalized Fourier transform and its applications, Integral Transforms Spec. Funct. 13 (2002), 321-344.

[37] Watanabe S., An embedding theorem of Sobolev type, Integral Transforms Spec. Funct. 15 (2004), 369-374.

[38] Witten E., Supersymmetry and Morse theory, J. Differential Geom. 17 (1982), 661-692.

[39] Yang L.M., A note on the quantum rule of the harmonic oscillator, Phys. Rev. 84 (1951), 788-790. 